\title{
Droperidol $v$. haloperidol for sedation of aggressive behaviour in acute mental health: randomised controlled trial
}

\author{
Leonie Calver, Vincent Drinkwater, Rahul Gupta, Colin B. Page and Geoffrey K. Isbister
}

\section{Background}

Agitation and aggression are significant problems in acute psychiatric units. There is little consensus on which drug is most effective and safest for sedation of these patients.

\section{Aims}

To compare the effectiveness and safety of haloperidol $v$. droperidol for patients with agitation and aggression.

\section{Method}

In a masked, randomised controlled trial (ACTRN12611000565943) intramuscular droperidol (10 mg) was compared with intramuscular haloperidol (10 mg) for adult patients with acute behavioural disturbance in a psychiatric intensive care unit. The primary outcome was time to sedation within $120 \mathrm{~min}$. Secondary outcomes were use of additional sedation, adverse events and staff injuries.

\section{Results}

From 584 patients, 110 were randomised to haloperidol and 118 to droperidol. Effective sedation occurred in 210 (92\%) patients within $120 \mathrm{~min}$. There was no significant difference in median time to sedation: 20 min (interquartile range $15-30$, range $10-75)$ for haloperidol $V .25 \mathrm{~min}$ (IQR 15-30, range 10-115) for droperidol $(P=0.89)$. Additional sedation was used more often with haloperidol (13\% v. 5\%, $P=0.06$ ), but adverse effects were less common with haloperidol ( $1 \%$ v. $5 \%, P=0.12)$. There were 8 staff injuries.

\section{Conclusions}

Both haloperidol and droperidol were effective for sedation of patients with acute behavioural disturbance.

\section{Declaration of interest}

None.
Acute behavioural disturbance including verbal aggression and violence is a common occurrence in acute mental health units, with the majority of cases resulting from acute psychosis or substance misuse. ${ }^{1,2}$ Most patients settle with verbal de-escalation or agree to take oral medication. ${ }^{2}$ Such approaches fail in a proportion of patients whose behaviour escalates from verbal abuse to physical violence. These people often require physical restraint and involuntary parenteral sedation, to prevent harm to other patients, staff and property. The aim in these cases is for the patient to be rapidly tranquillised or sedated. ${ }^{2,3}$ Despite the extent of the problem there is little consensus on the most effective and safest drugs for sedation, and there is limited evidence. ${ }^{4}$ Clinical practice is generally guided by individual or institutional experience. Guidelines recommend an antipsychotic or benzodiazepine as a single agent or in combination, or an antipsychotic with promethazine. ${ }^{2,5,6}$ Common choices are haloperidol alone; haloperidol and lorazepam; haloperidol with promethazine; or a newer antipsychotic such as olanzapine. ${ }^{3,5,7}$ Droperidol has been used less commonly after a Food and Drug Administration (FDA) boxed warning in 2002, but recently has been shown to be effective and safe in studies in emergency departments. ${ }^{8,9}$ Benzodiazepines are problematic in non-critical care settings because they may cause respiratory depression, and a recent systematic review found that adding a benzodiazepine to haloperidol afforded no benefit but carried the risk of harm. ${ }^{4}$ The same review concluded that independent trials with simple outcomes are required to improve the evidence for rapid tranquillisation, because there are significant risks associated with many recommended drugs. ${ }^{4}$ We aimed to compare the effectiveness and safety of droperidol with haloperidol for the sedation of patients with acute behavioural disturbance in an acute mental health unit.

\section{Method}

We undertook a masked, randomised controlled study of intramuscular haloperidol $(10 \mathrm{mg}) v$. intramuscular droperidol $(10 \mathrm{mg})$ for the rapid tranquillisation of patients with acute behavioural disturbance in a psychiatric intensive care unit. The primary outcome was the time to sedation. The study was undertaken from August 2011 to June 2013 in the psychiatric intensive care unit of a large tertiary specialist mental health facility in Australia. The psychiatric emergency care centre of this facility receives over 4400 presentations per year. Patients are generally referred from general practitioners, regional hospitals, other units within the institution or from the community usually by the community mental health teams or the ambulance or police service. Ethical approval was obtained from the local human research ethics committee. Consent was waived because of patients' lack of decision-making capacity to consent to medical treatment as duty of care. The trial was registered with the Australian New Zealand Clinical Trial Registry (ACTRN12611000565943).

\section{Study participants}

All patients with agitation or aggression who were admitted involuntarily to the psychiatric intensive care unit from the psychiatric emergency care centre were eligible for inclusion in the study. They were managed according to a standardised sedation protocol including the use of a purpose-designed acute behavioural disturbance chart. Participants were all adults ( $>18$ years of age) with acute behavioural disturbance who required parenteral medication for sedation and in whom verbal 
de-escalation and/or oral medication had failed. We excluded patients willing to take oral medication for sedation without physical restraint or seclusion and patients under 18 years old.

\section{Interventions}

Patients were identified for the study from either the psychiatric emergency care centre or the in-patient psychiatric intensive care unit. Once patients were recruited to the study they were escorted to the psychiatric intensive care unit and physically restrained with the assistance of security staff to allow the administration of intramuscular medication to the gluteal region. Patients were then either taken to their own room or placed in a seclusion room. They were not physically restrained once the medication had been administered. There was access to resuscitation equipment at all times, and staff had regular training in basic cardiopulmonary resuscitation. The psychiatric intensive care unit is staffed with four nurses for eight patients during daylight hours and the evening, and three nurses overnight. A psychiatrist and a psychiatric registrar are on site in working hours, and a medical officer is available in the hospital after hours.

Pre-packed treatment kits were available in the psychiatric intensive care unit; these had been produced by the Calvary Mater Newcastle pharmacy in conjunction with Richard Stenlake Compounding Chemist, Sydney, Australia. Each kit contained either droperidol $(10 \mathrm{mg}$ in $2 \mathrm{ml})$ or haloperidol $(10 \mathrm{mg}$ in $2 \mathrm{ml}$ ). Droperidol was purchased from Phebra Ltd (Sydney, Australia); the haloperidol was purchased from Fagron Ltd (Sydney, Australia) and transferred into vials identical to those containing the droperidol formulation. This was done under aseptic conditions by Richard Stenlake Compounding Pharmacy. The $10 \mathrm{mg}$ droperidol dose was based on a similar study in the emergency department and $10 \mathrm{mg}$ of both droperidol and haloperidol are equivalent doses used in rapid tranquillisation in psychiatry.

Block randomisation was used. Microsoft Excel was used to randomly create blocks of four ( $\mathrm{ABAB}, \mathrm{AABB}$, etc.) or six (ABABAB, AAABBB, etc.). The use of different block sizes meant that it was impossible to predict the next treatment. Each A or B allocation was then assigned a study code. The list of study codes with allocations was generated by a research assistant and supplied to the Calvary Mater Newcastle pharmacy, so that the investigators and treating staff remained unaware of the allocations. The pharmacy relabelled the vials of haloperidol or droperidol with study numbers based on the list of allocations. The vials were then supplied to the psychiatric intensive care unit in sequential order. The psychiatric intensive care unit was kept stocked with treatment kits for the duration of the study. Patients were administered the trial drug and then observed in the seclusion room. Vital signs and the level of agitation and sedation were recorded at $10 \mathrm{~min}$ intervals after the trial drug for at least $1 \mathrm{~h}$ or until the patient settled. Additional sedation was recommended if the patient showed no sign of settling $30 \mathrm{~min}$ after the initial sedation, but this was given at the discretion of the treating physician.

\section{Data collection and processing}

A previously developed acute behavioural disturbance chart was introduced into the psychiatric intensive care unit 1 year prior to the trial commencing. ${ }^{10}$ During this introductory year the chart was used to record prospectively the level of agitation and sedation in all patients, using the Sedation Assessment Tool (SAT). ${ }^{11}$ The SAT (see Appendix) scores the patient from +3 (physically violent) to -3 (unconscious) and allows rapid assessment before and after sedative medication is given. This initial year familiarised the staff with scoring the SAT, developing confidence in its utility and reliability, and ensured that both SAT scores and vital signs were recorded in all patients correctly for the trial. ${ }^{12}$ A baseline SAT score was recorded when the patient was recruited to the study; SAT scores and vital signs were then recorded every $10 \mathrm{~min}$ after the trial drug for the first hour, then half-hourly until the patient settled. A number of the observations were initially recorded remotely from outside the seclusion room, including respiratory rate and SAT score. Remote observations were commenced from the onset of the acute behavioural disturbance until it was considered safe to approach the patient and record vital signs including heart rate, blood pressure, oxygen saturation and respiratory rate. Adverse events and staff injury were recorded along with any observed extrapyramidal side-effects. Additional medications were given at the discretion of the treating doctors, recorded on the data sheet and charted on the medication chart.

\section{Outcome measures}

The primary outcome was the time to sedation, defined as time from the administration of the trial drug until the SAT score decreased by 2 or more or the score was 0 (calm and alert). ${ }^{11,12}$ Failed sedation was defined as the patient not being sedated within $120 \mathrm{~min}$. Adverse drug effects were defined as a respiratory rate less than 12 breaths/min, systolic blood pressure less than $90 \mathrm{mmHg}$, heart rate less than 60 beats/min, oxygen saturation less than $90 \%$ or the presence of extrapyramidal side-effects. The use of additional sedation was any medication administered within $60 \mathrm{~min}$ of the time of the study drug being given. Successful sedation was defined post hoc as patients sedated within $120 \mathrm{~min}$ who did not require additional sedation and had no adverse effects.

\section{Statistical analysis}

The sample size was calculated to be 230 so as to detect a difference in the time to sedation of $20 \mathrm{~min}$ between groups, assuming a within-group standard deviation of $30 \mathrm{~min}$ (based on a retrospective audit of psychiatric intensive care unit patients). Because time to sedation was likely to be a non-parametric continuous variable the sample size was calculated using the $t$-test $(\alpha=0.01, \beta=0.9)$ and $15 \%$ added. At the completion of the study one investigator (G.I.) still masked to the allocation audited all primary and secondary outcomes using the original data sheets. Another investigator not involved in recruiting patients or coordination of the study (C.P.) was then given the masked data, and separately the group allocations as either A or B by the pharmacy. At this time only the study labels A or B and not the drug names were known to the investigator. This investigator analysed the data independently and presented this to the other investigators. Only then did the pharmacy reveal whether A or B was haloperidol or droperidol.

Medians, interquartile ranges (IQRs) and ranges are reported for continuous variables. Percentages are reported for dichotomous outcomes with $95 \%$ confidence intervals. The continuous primary outcome was analysed using the MannWhitney test because the data were non-parametric. Dichotomous secondary outcomes were analysed using a two-tailed Fisher's exact test. A significance level of $P<0.05$ was used. All analyses and graphics used GraphPad Prism version 6.02 for Windows (www.graphpad.com).

\section{Results}

There were 584 sedation episodes during the 23-month study period and of these 356 were not included in the analysis because the treating clinician elected to give labelled parenteral sedation 


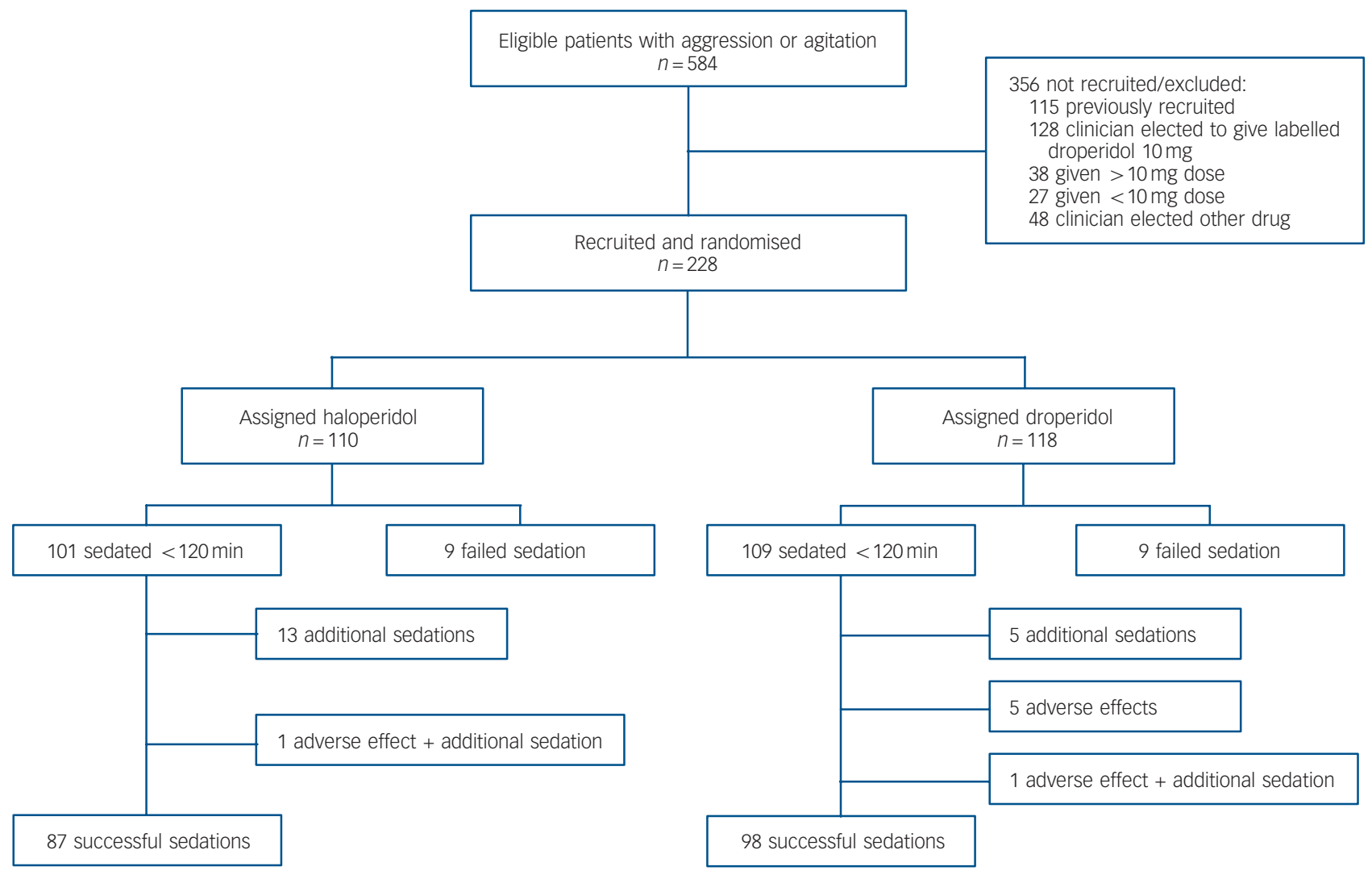

Fig. 1 Patient recruitment and allocation to haloperidol or droperidol.

(most commonly droperidol $10 \mathrm{mg}$ ) or a lower or higher dose of labelled parenteral medication, or it was recognised that the patient had already been recruited to the study (Fig. 1). An acute behavioural disturbance chart was filled out for 165 of these 356 episodes (46\%), and the baseline characteristics of the patients did not differ from those recruited to the study (Table 1). In 112 of the 356 episodes (31\%) an initial SAT score was recorded which was similar to those of the study participants (Table 1).

There were 228 episodes of acute behavioural disturbance in 206 patients recruited to the study, with 5 being sedated on three occasions, 12 on two occasions and 189 on one occasion. In all 228 episodes the patients were randomised: 110 were allocated haloperidol and 118 allocated droperidol. The median age was 33 years (range 16-71, IQR 27-43) and 144 were male (63\%). All were involuntary patients; $114(50 \%)$ had a primary diagnosis of mental illness and $70(31 \%)$ had been admitted with acute behavioural disturbance due to psychostimulant drugs. Baseline SAT scores were +3 in 111 episodes (49\%), +2 in 113 episodes $(50 \%),+1$ in 3 episodes and not recorded in one. One hundred and ten patients entered into the study needed to be placed in the seclusion room for the protection of themselves and others. Demographic data, cause of acute behavioural disturbance and baseline SAT scores were similar in the treatment groups except that more patients with primary mental illness received haloperidol

\begin{tabular}{|c|c|c|c|}
\hline & $\begin{array}{l}\text { Haloperidol group } \\
\qquad n=110\end{array}$ & $\begin{array}{l}\text { Droperidol group } \\
\qquad n=118\end{array}$ & $\begin{array}{l}\text { Excluded or not recruited } \\
\qquad n=356\end{array}$ \\
\hline Age, years: median (IQR) & $34(28-44)$ & $33(23-42)$ & $38(28-50)$ \\
\hline Gender (male), $n$ (\%) & $69(63)$ & $75(64)$ & $192(54)$ \\
\hline \multicolumn{4}{|l|}{ Presenting complaint, $n(\%)$} \\
\hline Mental illness & $62(56)$ & $52(44)$ & $222(62)$ \\
\hline Drug-induced psychosis & $30(27)$ & 40 (34) & 59 (17) \\
\hline Intoxication & $6(5)$ & $11(9)$ & $16(5)$ \\
\hline Threatened self-harm & 4 (4) & $2(2)$ & $54(15)$ \\
\hline Other/unknown & $6(5)$ & $13(11)$ & \\
\hline \multicolumn{4}{|l|}{ Baseline SAT score, $n$ (\%) } \\
\hline+3 & $50(45)$ & $61(52)$ & $77(50)^{\mathrm{a}}$ \\
\hline+2 & $56(51)$ & $57(48)$ & $72(47)$ \\
\hline+1 & $3(3)$ & $0(0)$ & $5(3)$ \\
\hline Prior sedation, $n(\%)$ & 9 (8) & $11(9)$ & NA \\
\hline Midazolam given with trial drug, $n(\%)$ & $2(2)$ & 7 (6) & NA \\
\hline
\end{tabular}




\begin{tabular}{|c|c|c|c|}
\hline & $\begin{array}{l}\text { Haloperidol group } \\
\qquad n=110\end{array}$ & $\begin{array}{l}\text { Droperidol group } \\
\qquad n=118\end{array}$ & $\begin{array}{l}\text { Excluded or not recruited } \\
\qquad n=356\end{array}$ \\
\hline Time to sedation, $\min (\mathrm{IQR})$ & $20(15-30)$ & $25(15-30)$ & $20(20-30)$ \\
\hline Sedated within $120 \mathrm{~min}, n$ (\%) & $101(92)$ & $109(92)$ & \\
\hline Additional sedation, $n$ (\%) & $14(13)$ & $6(5)$ & \\
\hline \multicolumn{4}{|l|}{ Adverse effects, $n(\%)$} \\
\hline Hypotension & $1(1)$ & $3(3)$ & \\
\hline Hypotension/desaturation & $0(0)$ & $1(1)$ & \\
\hline Extrapyramidal side-effects & $0(0)$ & 1 (1) & \\
\hline Oversedation & $0(0)$ & $1(1)$ & \\
\hline Staff injuries, $n$ (\%) & $5(5)$ & $3(3)$ & \\
\hline Midazolam given with trial drug, $n$ (\%) & $2(2)$ & 7 (6) & NA \\
\hline
\end{tabular}

and more with psychostimulant effects received droperidol (Table 1). In breach of the study protocol midazolam was given nine times simultaneously with the study drug, twice in the haloperidol group and seven times in the droperidol group.

\section{Primary outcome}

Effective sedation was achieved in 210 of 228 episodes (92\%) with 9 patients receiving haloperidol and 9 patients receiving droperidol not sedating within $120 \mathrm{~min}$ (Table 2). The median time to sedation was $20 \mathrm{~min}$ (IQR 15-30, range 10-75) for haloperidol compared with $25 \mathrm{~min}$ (IQR 15-30, range 10-115), which was not statistically significantly different $(P=0.89)$ (Figs 2,3$)$. The median time to sedation in 126 of the 356 episodes not included in the trial was $20 \mathrm{~min}$ (IQR 20-30, range 10-70).

\section{Secondary outcomes}

Additional sedation was required in 20 of 228 episodes: 14 (13\%) after haloperidol was given and $6(5 \%)$ after droperidol was given (difference $7.6 \%, 95 \%$ CI $0.3-15 ; P=0.059$ ). Three of the 18 patients (17\%) not sedated were given additional sedation. Adverse effects occurred in seven episodes (3\%), one in the haloperidol group and six in the droperidol group: 1 of 110 (1\%) $v .6$ of $118(5 \%) ; P=0.12$ (see Table 2). Staff injuries resulted from assaults prior to the administration of parenteral sedation and often occurred while the patient was being restrained. There were 44 staff injuries due to acute behavioural disturbance in the psychiatric intensive care unit during the 2-year study period and only eight were recorded on the study data sheets.

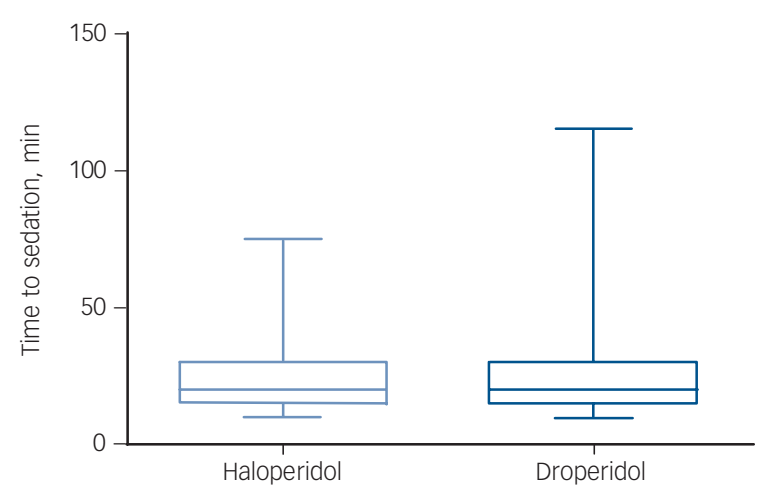

Fig. 2 Time to sedation for intramuscular haloperidol (10 mg) v. droperidol (10 mg).

\section{Discussion}

We found droperidol and haloperidol to be equally effective for the sedation of patients with acute behavioural disturbance in the acute mental health setting. The time to sedation for both drugs was similar and an equal proportion of patients were sedated within $120 \mathrm{~min}$ (92\%) with a median time to sedation of $20-25 \mathrm{~min}$. Although not statistically significant, there were more adverse effects in the patients given droperidol, mainly hypotension, but in no case was treatment required. Conversely, more additional sedation was required in patients given haloperidol, which again was not a statistically significant finding. The study suggests that either haloperidol or droperidol is suitable for the rapid tranquillisation of agitated and aggressive patients in an acute psychiatric unit, half of whom had a primary mental illness.

The most commonly used and recommended drugs for acute behavioural disturbance are benzodiazepines and antipsychotics. ${ }^{6}$ A recent study reviewing the current trends in the UK reported lorazepam as the most recommended medication followed by haloperidol, and concluded that multiple agents and combinations are commonly used. ${ }^{13}$ In contrast to clinical practice, the systematic review by Powney et al found limited evidence to support the use of haloperidol alone, better evidence to support haloperidol with promethazine (to decrease the rate of extrapyramidal side-effects) and no evidence to support the combination of haloperidol and benzodiazepines - and probable harm in the latter combination. ${ }^{4}$ In addition, no study has found that other antipsychotics are superior to haloperidol. ${ }^{4}$ Our study adds to this and provides further evidence that haloperidol alone is effective and that droperidol is similar and not more effective.

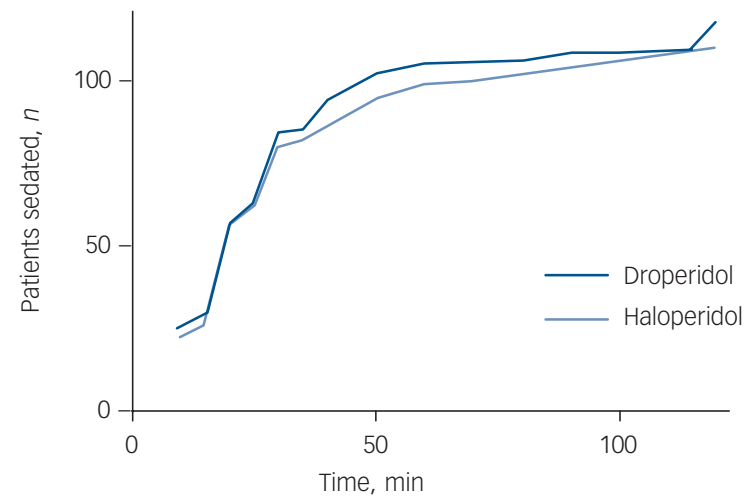

Fig. 3 Cumulative proportion of patients sedated $v$. time after drug administration 


\section{Previous studies}

Only two small trials have previously directly compared droperidol with haloperidol, one in the emergency department and one in an acute psychiatric unit. ${ }^{14,15}$ Both trials found that intramuscular droperidol required less additional sedation compared with haloperidol, but were small and of low quality. ${ }^{4,14,15}$ There has been a resurgence of the use of droperidol in Australia in the past few years owing to its success for sedation of violent and agitated patients in the emergency department. ${ }^{8,9,16,17}$ The validity of the 'black box' warning has been questioned by a systematic review, ${ }^{18}$ and there are large studies of the safe use of droperidol for sedation prior to the warning. ${ }^{19}$ This has, in turn, increased the use of droperidol in some mental health units. Haloperidol has remained the major conventional antipsychotic used to treat undifferentiated acute behavioural disturbance in acute psychiatric units. It has been the most studied antipsychotic and has been used as a comparator in countless trials in mental health. ${ }^{4}$ Haloperidol has been shown to be as effective as atypical antipsychotics, ${ }^{20}$ but has a higher propensity for extrapyramidal side-effects and has a well-defined association with QT prolongation and torsades de pointes in large doses. ${ }^{21,22}$

There are numerous studies of sedation or tranquillisation of patients with agitation or aggressive behaviour in the mental health setting, but few of these focus on highly agitated and aggressive patients who require physical restraint and parenteral sedation. For such patients rapid sedation rather than 'tranquillisation' is required, which means patients need to be contained within 30-60 min. Most studies in the mental health setting have outcomes at $2 \mathrm{~h}, 4 \mathrm{~h}, 6 \mathrm{~h}$ and $24 \mathrm{~h},{ }^{23-25}$ which are clearly not appropriate for this type of patient. In contrast, a previous study of haloperidol used a primary outcome of sedation within 20 min and had similar times to sedation to our study. ${ }^{3}$ In addition, many other studies exclude intoxicated patients and those with substance misuse, ${ }^{24,25}$ making generalising their results to clinical practice difficult because drug and alcohol use and intoxication are common in acute mental health admissions. Our study was restricted to a population of patients with severe agitation and aggression that required parenteral medication, and did not exclude any patient with a drug and alcohol history, making it more applicable to clinical practice.

Undertaking randomised controlled trials in this cohort of patients is difficult for many reasons, not least being the ethical issues surrounding consent for research involving these people. We have demonstrated in Australia that it is possible to undertake a controlled trial of medication without consent in this patient group. ${ }^{23}$ The local human research ethics committee agreed parenteral sedation with physical restraint was already being used without patient consent as a duty of care for treatment of these patients. The committee therefore allowed us to waive consent for a study that compared two treatments that were already given as part of standard clinical care. Most controlled trials have required consent for patient recruitment, ${ }^{24-28}$ which has meant that the trials excluded the majority of severely agitated and violent patients, who were included in our study. Such studies are less useful for defining treatment in patients with severe acute behavioural disturbance, whereas our study provides important evidence about commonly used drugs to inform clinical guidelines for these high-risk and difficult to manage patients.

\section{Adverse events}

The large number of staff injuries reported over the study period confirms the severity of the behaviour in these patients. However, all injuries to staff were from assaults prior to parenteral sedation or were sustained in the process of restraining the patient. This

suggests that the rapid parenteral sedation of these patients with haloperidol or droperidol prevented further injury to staff. The strict monitoring of adverse effects in this study allowed accurate assessment during the initial period following the onset of sedation. The most common adverse effect was hypotension, which was transient and did not require intervention. Hypotension occurred more commonly with droperidol. It is therefore important that there is routine assessment of blood pressure in patients given droperidol when it is safe to do so. Both droperidol and haloperidol are known to cause extrapyramidal side-effects but in this study there was only one episode of dystonia reported which quickly resolved with oral benztropine.

\section{Limitations}

A potential limitation of our study was the number of episodes where patients were not eligible to be recruited, which may have resulted in selection bias. In almost a third of these epsodes the patient was excluded from the study because they had already been recruited. The remaining patients were not recruited because of the clinicians' preference for a particular agent, a different dose of the drugs or a combination of drugs. However, the baseline characteristics of excluded patients were no different from those recruited to the study, including the baseline SAT scores. In addition, the excluded patients had a similar median time to sedation, suggesting that the use of higher doses and combinations was no better than haloperidol or droperidol alone. A major limitation of our study was that extrapyramidal side-effects might have occurred after the $120 \mathrm{~min}$ observation period. This is the most likely reason for the low rate of extrapyramidal side-effects reported, because such effects often occur many hours after the drug is administered. Numerous studies of haloperidol $v$. other drugs and/or placebo clearly show that extrapyramidal side-effects are more common with haloperidol. ${ }^{4}$ The difference in previous studies is that they were over longer periods and were therefore more likely to report extrapyramidal side-effects.

Finally, the study was not powered to detect differences in the secondary outcomes including adverse effects and additional sedation. Larger studies are now required to determine whether droperidol or haloperidol are associated with a greater risk of adverse effects or requirement for additional sedation.

\section{Leonie Calver, School of Medicine and Public Health, University of Newcastle, New South Wales; Vincent Drinkwater, Rahul Gupta, MBBS, Psychiatric Emergency Service, Hunter New England Mental Health Service, New South Wales; Colin B. Page, MBChB, School of Medicine, University of Queensland, Brisbane; Geoffrey K. Isbister, MD, School of Medicine and Public Health, University of Newcastle, New South Wales, Australia}

Correspondence: Geoff Isbister, c/o Calvary Mater Newcastle, Edith Street, Waratah, NSW 2298, Australia. Email: geoff.isbister@gmail.com

First received 20 Apr 2014, final revision 16 Jul 2014, accepted 31 Jul 2014

\section{Funding}

G.K.I. is supported by a National Health and Medical Research Council Senior Research Fellowship (ID1061041).

\section{Appendix}

\section{Sedation Assessment Tool}

Score Responsiveness

+3 Combative, violent, out of control

+2 Very anxious and agitated

$+1 \quad$ Anxious/restless

0 Awake and calm/cooperative

$-1 \quad$ Asleep but rouses if name is called

-2 Responds to physical stimulation

-3 No response to stimulation

\section{Speech}

Continual loud outbursts

Loud outbursts Normal/talkative Speaks normally

Slurring or prominent slowing Few recognisable words None 


\section{References}

1 Atakan Z, Davies T. ABC of mental health. Mental health emergencies. BMJ 1997; 314: 1740-2.

2 McAllister-Williams RH, Ferrier IN. Rapid tranquillisation: time for a reappraisal of options for parenteral therapy. Br J Psychiatry 2002; 180: 485-9.

3 Huf G, Coutinho ES, Adams CE. Rapid tranquillisation in psychiatric emergency settings in Brazil: pragmatic randomised controlled trial of intramuscular haloperidol versus intramuscular haloperidol plus promethazine. BMJ 2007; 335: 869 .

4 Powney MJ, Adams CE, Jones H. Haloperidol for psychosis-induced aggression or agitation (rapid tranquillisation). Cochrane Database Syst Rev 2012; 11: CD009377

5 National Institute for Health and Care Excellence. Violence: The Short-term Management of Disturbed/Violent Behaviour in In-patient Psychiatric Settings and Emergency Departments. Clinical Guideline CG25. NICE, 2005.

6 Lepping P. The use of emergency psychiatric medication: a survey from 21 countries. J Clin Psychopharmacol 2013; 33: 240-2.

7 Pilowsky LS, Ring $\mathrm{H}$, Shine PJ, Battersby M, Lader M. Rapid tranquillisation. A survey of emergency prescribing in a general psychiatric hospital. Br J Psychiatry 1992; 160: 831-5.

8 Isbister GK, Calver LA, Page CB, Stokes B, Bryant JL, Downes MA. Randomized controlled trial of intramuscular droperidol versus midazolam for violence and acute behavioral disturbance: the DORM study. Ann Emerg Med 2010; 56: 392-401 e1.

9 Knott JC, Taylor DM, Castle DJ. Randomized clinical trial comparing intravenous midazolam and droperidol for sedation of the acutely agitated patient in the emergency department. Ann Emerg Med 2006 47: 61-7.

10 Calver LA, Downes MA, Page CB, Bryant JL, Isbister GK. The impact of a standardised intramuscular sedation protocol for acute behavioural disturbance in the emergency department. BMC Emerg Med 2010; 10: 14.

11 Calver LA, Stokes B, Isbister GK. Sedation assessment tool to score acute behavioural disturbance in the emergency department. Emerg Med Australas 2011; 23: 732-40.

12 Calver L, Drinkwater V, Isbister GK. A prospective study of high dose sedation for rapid tranquilisation of acute behavioural disturbance in an acute mental health unit. BMC PSychiatry 2013; 13: 225.

13 Innes J, Sethi F. Current rapid tranquillisation documents in the UK: a review of the drugs recommended, their routes of administration and clinical parameters influencing their use. J Psychiatr Intens Care 2013; 9: 8.

14 Thomas $\mathrm{H}$, Schwartz $\mathrm{E}$, Petrilli R. Droperidol versus haloperidol for chemical restraint of agitated and combative patients. Ann Emerg Med 1992; 21: 407-13.
15 Resnick M, Burton BT. Droperidol vs. haloperidol in the initial management of acutely agitated patients. J Clin Psychiatry 1984; 45: 298-9.

16 Richards JR, Derlet RW, Duncan DR. Chemical restraint for the agitated patient in the emergency department: lorazepam versus droperidol. J Emerg Med 1998; 16: 567-73.

17 Martel M, Sterzinger A, Miner J, Clinton J, Biros M. Management of acute undifferentiated agitation in the emergency department: a randomized double-blind trial of droperidol, ziprasidone, and midazolam. Acad Emerg Med 2005; 12: 1167-72.

18 Kao LW, Kirk MA, Evers SJ, Rosenfeld SH. Droperidol, QT prolongation, and sudden death: what is the evidence? Ann Emerg Med 2003; 41: 546-58.

19 Chase $\mathrm{PB}, \mathrm{Biros} \mathrm{MH}$. A retrospective review of the use and safety of droperidol in a large, high-risk, inner-city emergency department patient population. Acad Emerg Med 2002; 9: 1402-10.

20 Wang HR, Woo YS, Bahk WM. Atypical antipsychotics in the treatment of delirium. Psychiatry Clin Neurosci 2013; 67: 323-31.

21 Sharma ND, Rosman HS, Padhi ID, Tisdale JE. Torsades de Pointes associated with intravenous haloperidol in critically ill patients. Am J Cardiol 1998; 81: 238-40.

22 Muzyk AJ, Rayfield A, Revollo JY, Heinz H, Gagliardi JP. Examination of baseline risk factors for QTC interval prolongation in patients prescribed intravenous haloperidol. Drug Saf 2012; 35: 547-53.

23 Wilhelm S, Schacht A, Wagner T. Use of antipsychotics and benzodiazepines in patients with psychiatric emergencies: results of an observational trial. BMC Psychiatry 2008; 8: 61.

24 Meehan K, Zhang F, David S, Tohen M, Janicak P, Small J, et al. A doubleblind, randomized comparison of the efficacy and safety of intramuscular injections of olanzapine, lorazepam, or placebo in treating acutely agitated patients diagnosed with bipolar mania. J Clin Psychopharmacol 2001; 21: 389-97.

25 Meehan KM, Wang H, David SR, Nisivoccia JR, Jones B, Beasley CM, et al. Comparison of rapidly acting intramuscular olanzapine, lorazepam, and placebo: a double-blind, randomized study in acutely agitated patients with dementia. Neuropsychopharmacology 2002; 26: 494-504.

26 Lesem MD, Tran-Johnson TK, Riesenberg RA, Feifel D, Allen MH, Fishman R, et al. Rapid acute treatment of agitation in individuals with schizophrenia: multicentre, randomised, placebo-controlled study of inhaled loxapine. Br J Psychiatry 2011; 198: 51-8.

27 Wright P, Birkett M, David SR, Meehan K, Ferchland I, Alaka KJ, et al. Double-blind, placebo-controlled comparison of intramuscular olanzapine and intramuscular haloperidol in the treatment of acute agitation in schizophrenia. Am J Psychiatry 2001; 158: 1149-51.

28 Breier A, Meehan K, Birkett M, David S, Ferchland I, Sutton V, et al. A double-blind, placebo-controlled dose-response comparison of intramuscular olanzapine and haloperidol in the treatment of acute agitation in schizophrenia. Arch Gen Psychiatry 2002; 59: 441-8. 